\title{
MULTIWAVELENGTH STUDY OF THE SHAPLEY CONCENTRATION
}

\author{
S. BARDELLI ${ }^{1}$, E. ZUCCA ${ }^{2,3}$, G. ZAMORANI $^{2,3}$, \\ G. VETTOLANI ${ }^{3}$ AND R. SCARAMELLA ${ }^{4}$ \\ ${ }^{1}$ Osservatorio Astronomico di Trieste \\ ${ }^{2}$ Osservatorio Astronomico di Bologna \\ ${ }^{3}$ Istituto di Radioastronomia del CNR \\ ${ }^{4}$ Osservatorio Astronomico di Roma
}

\section{Introduction}

The Shapley Concentration is a prominent supercluster in the southern sky. It is interesting not only for its relevance in the peculiar motion problem (it seems to be responsible of $\sim 30 \%$ of the acceleration acting on the Local Group of galaxies), but also because it is the most remarkable feature which appears studying the distribution of the Abell-ACO clusters of galaxies: Zucca et al. (1993) found that at every density contrast the Shapley Concentration stands out as the richest supercluster in the sky. In particular, the central part of this supercluster is dominated by a complex containing the three ACO clusters A3556, A3558 and A3562 and the poor cluster SC1329-314, which form a structure elongated $\sim 3^{\circ}$ along the East-West direction. We are carrying on a long term study of the Shapley Concentration in order to describe its dynamical state and to determine its mass. The project consists of redshift determinations (with the ESO telescopes at La Silla) for galaxies both in the clusters (Bardelli et al. 1994) and in the inter-cluster field of this supercluster, X-ray observations (ROSAT) of the hot gas in the clusters (Bardelli et al. 1996) and radio observations (ATCA, MOST and VLA, Venturi et al. 1996) of the radiogalaxies. 


\section{The Inter-Cluster Survey}

The Shapley Concentration was discovered as a cluster structure, but nothing is known about the distribution and the overdensity of galaxies. In order to study the global dynamics of this supercluster, we started a redshift survey of galaxies in the central part of this structure and outside clusters or obvious bi-dimensional overdensities. We observed 26 fields obtaining 443 redshifts in the [17-18.8] magnitude range. The number of spectra which present emission lines is 74 , corresponding to $\sim 16 \%$, similar to the percentage found in clusters. In the field, the percentage is $\sim 44 \%$ in the same magnitude bin (Vettolani et al., this volume, p. 346). We found also that this structure is similar to the Great Wall, with a bridge of galaxies connecting the most massive clusters.

\section{The Core of the Shapley Concentration}

The central regions of rich superclusters could be considered as ideal laboratories for studying the dynamical phenomena related to the formation of rich galaxy clusters. The higher local density excesses lead to high peculiar velocities which, when added to the relative small volume involved, increase the "cross section" for collisions and mergings. The Shapley Concentration shows two main cluster complexes, signatures of ongoing merging processes. We studied the complex dominated by A3558 (Bardelli et al. 1994, 1997 in preparation) using a sample of 714 redshifts, finding that it is a single connected structure of strongly interacting clusters. We calculated the dynamical parameters for the clusters, finding the presence of a number of substructures, both in A3558 and A3556. In particular, two groups, located between A3562 and A3558, are very prominent both in the optical (Bardelli et al. 1994) and in the X-ray band (Bardelli et al. 1996). The galaxy distribution in the core of the Shapley Concentration resembles the results of Burns et al. (1993) for the case of a merging between two clusters. The knowledge of the overdensity of inter-cluster galaxies will permit us to estimate the relative impact velocity and to study the evolution of the structure. Moreover, our radio-optical analysis of the brightest objects of this structure will be useful for studying the influence on the physical properties of the galaxy population of a cluster-cluster merging process.

\section{References}

Bardelli S., Zucca E., Vettolani, G., et al., 1994, Mon.Not.R.astron.Soc. 267, 665

Bardelli S., Zucca E., Malizia A., et al., 1996, Astron.Astrophys. 305, 435

Burns J.O., Roettiger K., Ledlow M., Klypin A., 1993, Astrophys.J. 427, L87

Venturi, T., et al. 1996, Mon.Not.R.astron.Soc., in press

Zucca E., et al. 1993, Astrophys.J. 407, 470 